\title{
Perfil Antropométrico e Dietético de Crianças Expostas ao HIV
}

\section{Anthropometric and Dietary Profile of Children Exposed to HIV}

Millena Araujo de Souza1, Lúcia Helena Almeida Gratão², Renata Junqueira Pereira $^{3}$

\section{RESUMO}

Objetivo: Delinear o perfil antropométrico e dietético de crianças, expostas ao HIV/AIDS, acompanhadas em um serviço especializado, de Palmas, Tocantins. Método: Estudo transversal, com crianças de 0 e 18 meses, que tiveram os dados coletados dos prontuários e nas consultas de nutrição. Resultados: Foram estudadas 13 crianças, com idade média de 6,63 $\pm 3,74$ meses e $69,2 \%$ do sexo masculino. A maioria apresentouse eutrófica em relação aos índices peso para a idade, comprimento para a idade, índice de massa corporal para a idade, peso para o comprimento e perímetro cefálico para a idade ao nascer e atuais. A nenhuma das crianças foi ofertada alimentação complementar antes dos 4 meses de idade, porém a maioria está com o esquema alimentar inadequado para a fase de vida (92,3\%). A necessidade diária média de energia foi de $633,77 \mathrm{Kcal}$, a ingestão diária de energia média foi de $866,0 \mathrm{Kcal}$ e 0 percentual médio de adequação da ingestão à necessidade foi de $136,92 \% \%$. Os cuidadores eram as próprias mães, com idade média de 28,15anos e $61,5 \%$ como ensino médio completo. A renda familiar média foi de $\mathrm{R} \$ 1.190,69$ reais e a renda per capita variou entre $R \$ 41,00$ e $R \$ 640,00$. Conclusão: O perfil antropométrico das crianças apresenta-se adequado, porém, observam-se crianças em risco de sobrepeso, o que pode ser agravado pela ingestão energética superior à necessidade. Os cuidadores, no geral, são mães jovens de baixa escolaridade, baixa renda, o que denota uma população mais vulnerável a complicações relacionadas ao HIV.

Palavras-Chave: Estado Nutricional. HIV. Infância.

\section{ABSTRACT}

Objective: To outline the anthropometric and dietary profile of children exposed to HIV / AIDS, accompanied by a specialized service, from Palmas, Tocantins. Method: Crosssectional study with children aged 0 and 18 months, who had the data collected from the charts and in the nutrition clinics. Results: We studied 13 children, mean age of 6,63 $\pm 3,74$ months and $69.2 \%$ of males. The majority were eutrophic in relation to the $P / I$, $\mathrm{C} / \mathrm{I}, \mathrm{BMI} / \mathrm{I}, \mathrm{P} / \mathrm{C}$ and PC / I indices at birth and current. None of the children received complementary feeding before 4 months of age, but most of them had an inadequate diet for the life stage (92.3\%). The mean daily energy requirement was $633.77 \mathrm{Kcal}$, the average daily energy intake was $866.0 \mathrm{Kcal}$ and the average percentage of adequacy of intake to the need was $136.92 \%$. The caregivers were the mothers themselves, with Mean age of 28.15 years and $61.5 \%$ with complete secondary education. The average family income was $R \$ 1,190.69$ reais and the per capita income varied between $R \$ 41.00$ and $R \$ 640.00$. Conclusion: The anthropometric profile of the children is adequate, however, children at risk of overweight are observed, which can be aggravated by a higher intake of energy. Caregivers, in general, are young mothers of low schooling, low income, which denotes a population more vulnerable to complications related to HIV.

Keywords: Nutritional Status. HIV. Childhood. 


\section{INTRODUÇÄO}

A Síndrome da Imunodeficiência Adquirida (AIDS), sofreu mudanças em seu perfil epidemiológico ao longo das últimas três décadas (BRASIL, 2017). Na década de 80 era prevalente entre os homossexuais, e posteriormente passou por um processo de feminilização da doença, que resultou em dois aspectos importantes a serem ressaltados: a inclusão de um grupo específico no perfil da síndrome, a mulher, e a problemática da transmissão vertical do HIV/AIDS, incluindo um novo grupo populacional na epidemia, as crianças (GOMES; CABRAL; SCHILKOWSKY, 2004; CARVALHO; PICCININI, 2008).

$\mathrm{O}$ diagnóstico do HIV pode transformar profundamente a vida de uma criança, interferindo psicologicamente no convívio social e familiar, bem como no seu desenvolvimento fisiológico, ainda nos seus hábitos alimentares e na forma como os nutrientes serão utilizados no organismo (GOMES; CABRAL; SCHILKOWSKY, 2004).

A garantia de um tratamento que assegure o bem-estar à pessoa vivendo com HIV/AIDS, depende de uma equipe multidisciplinar que trabalhe em sintonia com tecnologias de cuidado adequadas e eficazes para cada indivíduo. O tratamento não deve limitar-se apenas ao atendimento médico, mas sim envolver uma equipe multidisciplinar (FILHO, 2004; BRASIL, 2015). O estado nutricional destaca-se como fator que interfere diretamente nas condições de vida de uma criança e, caso infectada, suas condições de saúde e nutricionais tendem mais facilmente ao desequilíbrio. Portanto, o delineamento do perfil nutricional dessas crianças, envolve procedimentos que vão além de uma avaliação básica, mas que incluem ainda dados fornecidos pelos demais profissionais da saúde, apontando-se, por exemplo, o prontuário médico, o histórico de cada criança, os dados relatados no momento da consulta, suas vontades e medidas antropométricas (GOMES; CABRAL; SCHILKOWSKY, 2004; TANAKA et al., 2015).

Nesse sentido, são recomendações do Fundo das Nações Unidas para a Infância (UNICEF) e da Organização Mundial de Saúde (OMS) que se desenvolvam e se implementem, políticas públicas de saúde, voltadas à alimentação de lactentes e crianças jovens, considerando-se a influência do HIV (WHO, 2003). Segundo o Ministério da Saúde (BRASIL, 2006) toda criança, nascida de mãe e/ou amamentada por mulher vivendo com HIV, é considerada exposta. No Tocantins, no período de 2007 a 2012, foram notificadas, aproximadamente, 140 crianças nessa situação (SESAU, 2012). 
Desta forma o presente trabalho teve como objetivo delinear o perfil antropométrico e dietético de crianças expostas ao HIV, acompanhadas em um serviço de atendimento especializado, do município de Palmas, Tocantins.

\section{METODOLOGIA}

Trata-se de um estudo quantitativo, de natureza exploratória e descritiva, desenvolvido no ambulatório de um serviço de atendimento especializado (SAE), instituição integrante do Sistema Único de Saúde (SUS), na cidade de Palmas, Tocantins. O SAE recebe pacientes de todas as localidades do Estado, sendo um centro de referência em infecções sexualmente transmissíveis (IST) e doenças tropicais (DT).

A população do estudo foi constituída pelo total das crianças acompanhadas no SAE $(n=19)$, com idades entre 0 e 18 meses. Porém, três dessas crianças possuíam idades entre 5 e 6 anos e não foram incluídas no estudo, por terem perfil nutricional muito diferente da faixa etária de 0 a 18 meses. Outras três crianças não compareceram ao atendimento agendado e, por esse motivo, não foram incluídas no estudo. Das 13 crianças incluídas no estudo, nenhuma possui diagnóstico de infecção pelo vírus HIV.

Os dados foram coletados por meio de análises dos prontuários e durante a consulta ambulatorial de nutrição, realizadas entre os meses de outubro e novembro de 2016. A coleta de dados foi orientada por um formulário estruturado, contendo as variáveis de estudo, relacionadas à criança e ao cuidador.

Em relação à criança investigou-se: a idade, o sexo, a idade gestacional ao nascer, o peso ao nascer (PN) e o peso atual (PA), o comprimento ao nascer (CN) e o atual (CA) e o perímetro cefálico ao nascer (PCn) e o atual (PCa). Foram considerados dados atuais, aqueles obtidos no dia da consulta ambulatorial. Foram avaliados também parâmetros dietéticos, como o tipo de substituto do leite materno utilizado, o uso de farináceos, a idade de introdução da alimentação complementar e aplicado inquérito dietético de Recordatório Alimentar de 24 horas, e avaliação do esquema alimentar. Foi investigado também o uso de medicamentos de terapia antirretroviral (TARV). Em relação ao cuidador coletou-se a idade, o grau de escolaridade, a renda familiar e o número de pessoas no domicilio para cálculo de renda per capita.

Quanto ao tratamento dos dados antropométricos, utilizaram-se as curvas de peso para idade $(P / I)$, peso para comprimento $(P / C)$, comprimento para idade $(C / I)$, índice de massa corporal para idade (IMC/I) e perímetro cefálico para idade (PC/I), propostas pela OMS (WHO, 2007). Para avaliação do Recordatório Alimentar de 24 horas foi utilizado o 
software Dietbox® (DIETBOX, 2015) e as necessidades energéticas foram calculadas individualmente, segundo o requerimento energético estimado (EER) (REZENDE; PEREIRA, 2014).

As análises dos dados foram realizadas, por meio de análises descritivas, no programa Statistical Package for Social Sciences (SPSS), versão 20.0 (SPSSI, 2011).

A presente pesquisa foi aprovada pelo Comitê de Ética em Pesquisa da Universidade Federal do Tocantins, sob o parecer número: 1.618.415.

\section{RESULTADOS}

Foram estudadas 13 crianças expostas ao HIV, com idade média de 6,63 $\pm 3,74$ meses e mediana de 8 meses. Destas, 69,2\% eram do sexo masculino e $30,8 \%$ do sexo feminino. A média de idade gestacional ao nascer foi de 38,58 $\pm 1,31$ semanas. A média de peso ao nascer foi de $3.215 \pm 462,77 \mathrm{~g}$ e a mediana foi de $3.360 \mathrm{~g}$. Em relação ao $\mathrm{CN}$, a média ficou em 49,77 $\pm 1,69 \mathrm{~cm}$ e a mediana em $50 \mathrm{~cm}$. O PCn médio foi de 35,25 $\pm 1,05$ $\mathrm{cm}$, com mediana de $35 \mathrm{~cm}$.

A análise dos índices antropométricos, disposta no gráfico 1, mostra a classificação do estado nutricional das crianças, onde a maioria apresentou-se eutrófica em relação aos índices IMC/I ao nascer $(\mathrm{n}=9)$ e $\mathrm{P} / \mathrm{C}$ ao nascer $(\mathrm{n}=10)$; adequadas quanto a $\mathrm{C} / \mathrm{I}$ ao nascer ( $n=13)$ e PC/I ao nascer ( $n=12)$; sendo que para o $P / I$ ao nascer houve $100 \%$ de adequação $(n=13)$.

Em relação aos índices antropométricos atuais, apresentados no gráfico 2, o estado nutricional da maioria apresentou-se em eutrofia, quanto aos parâmetros IMC/I atual $(n=9)$, $P / C$ atual $(n=9)$ e $P C / I$ atual $(n=12)$. Todas apresentaram $P / /$ e $C / I$ atuais adequados $(100 \%)$. 
Gráfico 1. Classificação do estado nutricional ao nascer, por índice antropométrico, das crianças expostas ao HIV, atendidas num Serviço de Atendimento Especializado (SAE), 2016, em Palmas- TO.

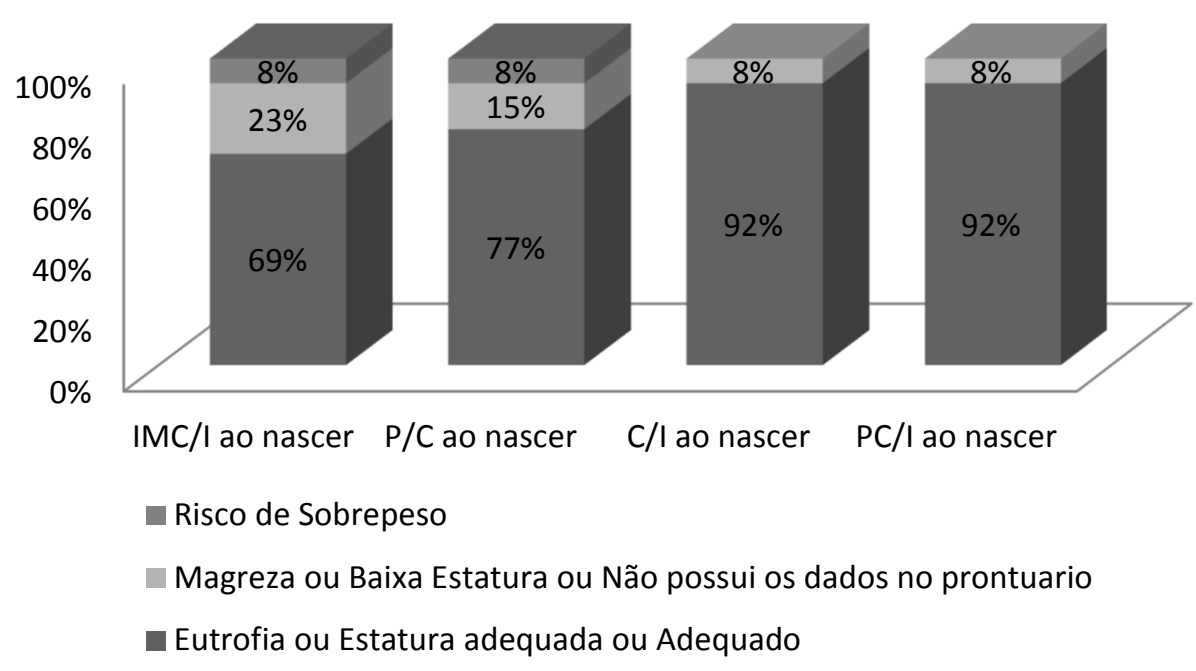

Gráfico 2. Classificação do estado nutricional atual, por índice antropométrico, de crianças expostas ao HIV, atendidas num Serviço de Atendimento Especializado (SAE), 2016, em Palmas- TO.

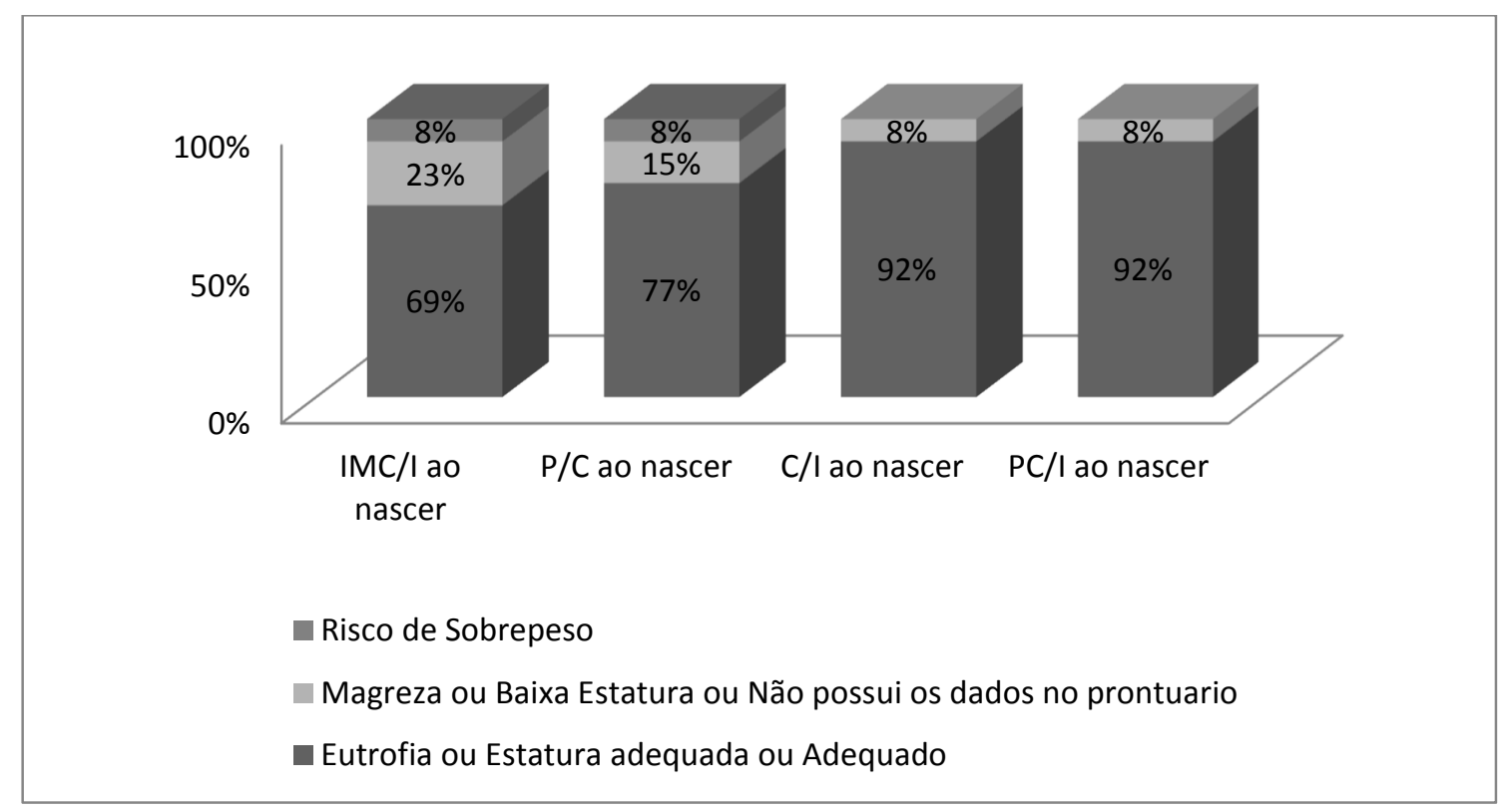

A análise do inquérito dietético do Recordatório Alimentar de 24 horas mostra que $69,2 \%$ das crianças estavam com ingestão energética superior à necessidade calculada para sua faixa etária pelo EER, enquanto apenas 30,8\% estavam com ingestão energética adequada. $O$ percentual de adequação da ingestão energética à recomendação variou 
entre 80 e 232\%. Na Tabela 1 estão dispostas as médias e medianas de ingestão e recomendação de energia e de adequação da ingestão energética ao EER calculado.

Tabela 1. Média, mediana e percentual de adequação da ingestão e da necessidade energética de crianças expostas ao HIV, atendidas num Serviço de Atendimento Especializado (SAE), 2016, em Palmas- TO.

\begin{tabular}{|c|c|c|c|c|c|}
\hline & Média & Desvio padrão & Mediana & Mínimo & Máximo \\
\hline IE (Kcal)* & 866,08 & $\pm 350,89$ & 760 & 356 & 1366 \\
\hline EER (Kcal)** & 633,77 & $\pm 139,56$ & 652 & 302 & 892 \\
\hline Adequação & 136,92 & $\pm 50,54$ & 125 & - & - \\
\hline
\end{tabular}

${ }^{*}$ Ingestão Energética

${ }^{* *}$ Requerimento Energético Estimado

Em relação à análise dietética, todas as crianças faziam uso de fórmula comercial, sendo que $46,2 \%$ consumiam fórmula comercial para lactentes (de 0 a 6 meses de idade), $46,2 \%$, fórmula de seguimento (a partir de 6 meses de idade) e 7,7\% consumiam fórmula para lactente, associada a leite de vaca integral. A nenhuma das crianças foi ofertada alimentação complementar, antes dos 4 meses de idade, porém a maioria está com o esquema alimentar inadequado para a fase de vida (92,3\%). Grande parte não consome farináceos $(76,9 \%)$, porem há consumo de alimentos industrializados (como bolacha salgada, iogurte adoçado), além da oferta de suco adoçado e baixo consumo de alimentos do grupo das carnes e leguminosas. Quanto ao uso de TARV, apenas 15,4\% utilizavam Zivodulina (AZT) no momento da avaliação, como medida profilática, seguindo o protocolo clínico para prevenção da infecção por transmissão vertical.

Em relação aos cuidadores, em sua totalidade esses eram as próprias mães das crianças, com idade média de 28,15 \pm 4,59 anos e mediana de 27 anos. Quanto ao grau de escolaridade, $61,5 \%$ das mães possuíam o ensino médio completo, $23,1 \%$ possuíam o ensino superior incompleto, 7,7\%, o ensino fundamental incompleto e 7,7\% possuíam o ensino médio incompleto. Ao analisar os dados de renda familiar, a média foi de $R \$$ $1.190,69$ reais $( \pm 713,60)$, pouco mais de 1 salário mínimo e a mediana foi de $R \$ 1.000,00$ reais. O número médio de pessoas por família foi de 4,5 . Ao se calcular a renda per capita, observou-se que variou entre $R \$ 41,00$ e $R \$ 640,00$, com média de $R \$ 273,30 \pm 157,38$ e mediana de $\mathrm{R} \$ 252,00$. 


\section{DISCUSSOEES}

As crianças selecionadas para esse estudo encontram-se em faixa etária de confirmação do diagnóstico de HIV/AIDS, uma vez que a doença pode se manifestar até os 18 meses.

As crianças do sexo masculino apresentam-se em maior percentual, assim como no estudo de Freitas et al. (2014). Quanto à idade gestacional ao nascer, segundo o Ministério da Saúde (2000), os recém-nascidos são classificados como a termo, quando nascem com idade gestacional entre 37 e 41 semanas e 6 dias. Assim, todas as crianças desse estudo nasceram a termo (BRASIL, 2000). De acordo com Tadielo et al. (2013), o risco de mortalidade de crianças nascidas a termo é bem menor, se comparadas a nascidos prétermo.

Quando se compara a idade gestacional ao nascer com o peso para idade e este se situa abaixo da normalidade (baixo peso, muito baixo peso e magreza), o risco de mortalidade também é muito elevado. Neste estudo, a maior parte das crianças nasceu com peso adequado para a idade e a termo, o que sinaliza menor risco de mortalidade.

A classificação do estado nutricional, considerando o padrão de referência proposto pela OMS (WHO, 2007), demonstra que, neste estudo, a maioria das crianças apresentou eutrofia ao nascer (Gráfico 1), independentemente do índice utilizado (todas próximas da mediana na curva). Entretanto, foi possível identificar que uma criança apresentou baixa estatura para a idade ao nascer, o que pode ter relação com a influência genética e/ou com influências do meio ambiente, dentre elas a alimentação materna, as condições socioeconômicas (AQUINO, 2011).

Um dado relevante foi observado em relação ao $\mathrm{MC} / \mathrm{l}$ ao nascer, onde $23 \%(\mathrm{n}=3)$ das crianças apresentaram magreza. As crianças expostas ou que tenham o diagnóstico de HIV/AIDS, frequentemente apresentam associação entre baixo peso e déficit de crescimento, o que aumenta a mortalidade infantil nessa população (DIAS et al., 2012).

Crianças expostas ao HIV necessitam de avaliação sistemática do crescimento e desenvolvimento, já que nos primeiros meses de vida podem apresentar sinais ou sintomas dos marcadores específicos do HIV, dentre eles alterações no perímetro cefálico (BRASIL, 2009). Afortunadamente, no presente estudo, todas as crianças que tiveram a medida PC/I disponível, apresentaram crescimento encefálico adequado para a idade.

Com relação ao estado nutricional atual das crianças (Gráfico 2), a classificação de risco de sobrepeso (23\%) para os índices IMC/I atual $(n=3)$ e P/C atual $(n=3)$, aponta para 
um aumento do risco de desenvolvimento de obesidade futura e das doenças e agravos não transmissíveis (DANT) associados ao excesso de peso. Quando se observa a tabela 1, nota-se ainda que a ingestão energética está muito acima da necessidade calculada, o que sinaliza para um risco de incremento dos índices de sobrepeso nessa população.

O uso de farináceos na alimentação de crianças é comum, dada a crença das mães de que o leite não supre todas as necessidades da criança, sendo necessário enriquecê-lo (BRASIL, 2002). Um estudo em São Paulo mostrou que açúcar e/ou espessantes, adicionados ao leite não materno, aumentaram significativamente a densidade energética da dieta (EGASHIRA et al., 1999). No presente estudo o percentual $(23,1 \%, n=3)$ encontrado de crianças consumindo farináceos é preocupante, visto que esse consumo pode levar ao aumento de peso e predispor ao risco de doenças e agravos não transmissíveis. Porém, dentre as crianças que se encontraram em risco de sobrepeso no presente estudo, não foi observado o consumo de farináceos.

O uso de farináceos como complemento da alimentação pode justificar a elevada prevalência de ingestão energética superior à necessidade. Todas as crianças em uso de farináceo no presente estudo estão consumindo mais energia que o calculado pelo EER. Para esse grupo que consome farináceos na alimentação, a necessidade diária média calculada de energia foi de $696 \pm 108,13 \mathrm{Kcal}$, enquanto a ingestão diária de energia média foi de 1261,67 $\pm 165,12$ Kcal e o percentual médio de adequação da ingestão à necessidade foi de $186,33 \pm 48,95 \%$.

A oferta de alimentação complementar, antes dos quatro meses de idade, pode ser extremamente prejudicial, principalmente a crianças que fazem o uso de fórmulas comerciais, pois a mucosa gastrointestinal ainda não está madura o suficiente para receber alimentos e o leite utilizado não possui os fatores de proteção do leite materno, o que pode desencadear doenças e infecções intestinais. Somado a isso, se a fórmula láctea ainda não for preparada sob condições adequadas de higiene, podem estar em grande vulnerabilidade e risco de infecções (BRASIL, 2002). No momento da coleta dos dados, existia um consenso no Ministério da Saúde (BRASIL, 2002; BRASIL, 2009) de que a introdução de alimentos complementares não deveria ser realizada antes dos quatro meses de idade. Neste estudo não se observou a oferta de alimentação complementar antes dos 4 meses de idade. No entanto, segundo a Sociedade Brasileira de Pediatria (2017) as crianças que fazem uso de fórmula infantil para lactentes, de forma exclusiva, também deverão iniciar a alimentação complementar aos 6 meses de idade. 
O esquema de alimentação complementar, proposto pelo Ministério da Saúde (BRASIL, 2004; BRASIL, 2009; BRASIL, 2015) para crianças que não podem ser amamentadas, preconiza que, do quarto ao oitavo mês, sejam ofertadas uma papa salgada, duas de frutas e três refeições lácteas acrescidas de cereal ou tubérculo; a partir do oitavo mês, deve-se iniciar a progressão da consistência alimentar e a introdução gradual da alimentação da família; para que, aos doze meses, a criança esteja com a alimentação consolidada. Analisando o padrão de consumo alimentar das crianças do presente estudo, observou-se que $92,3 \%$ das crianças não está seguindo o esquema alimentar proposto pelo MS, para a faixa etária na qual se encontra, principalmente pela ausência das duas papas de frutas diárias.

Sugere-se a adoção de políticas públicas no sentido de, não apenas disponibilizar substitutos do leite materno aos filhos de lactantes com HIV, mas também monitorar a oferta do substituto do leite e a introdução da alimentação complementar a essas crianças. Em relação aos alimentos que são ofertados, há introdução de industrializados (como bolacha salgada, iogurte adoçado), além da oferta de suco adoçado. Estes alimentos estão associados ao desinteresse por alimentos saudáveis e à formação de maus hábitos alimentares, associados a doenças carenciais como anemia ferropriva, à obesidade e às doenças crônicas. Quanto aos alimentos que devem compor a papa salgada (um alimento do grupo de cereais ou tubérculos, um de verduras ou legumes, um de leguminosas e um de carnes), observa-se que nenhuma das crianças estudadas consome papas salgadas em composição adequada (BRASIL, 2004; BRASIL, 2009; BRASL, 2015.

Em crianças com risco elevado de infecções, devido à exposição ao HIV, é preocupante quando a composição alimentar da dieta aumenta o risco de desenvolvimento de anemia. Isso se torna mais crítico quando se considera que a anemia ferropriva tem sua maior incidência nos dois primeiros anos de vida, podendo afetar o desenvolvimento cognitivo, o crescimento, a imunidade e a morbidade por infecções (SILVA, 2011).

Porém, os resultados do presente estudo apontam para aspectos preocupantes em relação ao perfil alimentar dessas crianças e sugere a realização de futuros estudos, com a utilização de ferramentas de coleta de dados dietéticos mais robustas.

Quanto ao esquema alimentar de oferta do leite artificial, o modo de preparo sugerido pelo fabricante em relação à diluição (uma colher medida nivelada de fórmula para cada 30 $\mathrm{mL}$ de água) e o número de mamadeiras a serem diariamente ofertadas para a faixa etária, não estão sendo corretamente aplicados pelas mães entrevistadas, sendo que a maioria 
delas utiliza diluição incorreta (acima ou abaixo do recomendado) ou número incorreto de mamadas (sempre acima do recomendado).

Segundo o Ministério da Saúde, a TARV deve ser iniciada em todos os recémnascidos expostos, de preferência ainda na sala de parto ou nas primeiras quatro horas de vida, durante quatro semanas (BRASIL, 2014). Neste estudo apenas duas crianças encontravam-se em uso de AZT, possivelmente devido a faixa etária no momento da coleta.

Nesse estudo a renda familiar média (1 salário mínimo) foi maior que a observada por Dias et al. (2010). A média de idade materna deste estudo foi semelhante à encontrada no estudo de Dias et al. (2010) e idêntica à encontrada por Galvão (2014). As condições de renda, idade materna e outros fatores sociais em que vivem as famílias com HIV, aumenta a vulnerabilidade das crianças às doenças próprias da infância, bem como o risco de atraso no crescimento e desenvolvimento (MACHADO et al., 2010).

Neste estudo o grau de escolaridade materna predominante foi o ensino médio completo (61,5\%), índice de escolaridade superior ao observados por Dias et al. (2010), em que a maioria das mães possuía apenas quatro anos de escolaridade formal (40\%), e por Freitas et al. (2014), que encontrou em sua amostra $32,2 \%$ das mães com menos de cinco anos de estudo formal.

O baixo grau de escolaridade materna representa um agravante à saúde das crianças, pois quanto menor o grau de escolaridade, mais dificuldades a mãe pode terpara cuidar do filho, pois geralmente não reconhece a importância da correta higiene ao manipular os alimentos, ou não consegue compreender corretamente os rótulos das fórmulas infantis comerciais, o que preocupa quando se trata de uma população de crianças com imaturidade imunológica, somada à exposição ao HIV (BARROSO et al., 2009; MACHADO et al., 2010; GALVÃO, 2014).

O acesso desta população aos serviços de saúde deve ser incentivado e fortalecido por meio da execução das políticas públicas atuais. Nesse sentido o Ministério da Saúde tem promovido ações de descentralização dos serviços especializados, levando-os para a atenção básica como meio de proporcionar melhor acesso aos pacientes vivendo com HIV/Aids e crianças expostas (GRANGEIRO, CASTANHEIRA E NEMES, 2015).

$\mathrm{Na}$ atenção básica o indivíduo pode ter acesso a uma equipe multiprofissional, que poderá proporcionar atendimento individual e/ou em grupo, adequado as suas necessidades (BRASIL, 2009). Entre os membros da equipe pode-se ter acesso ao nutricionista, que tem papel fundamental na melhoria da qualidade de vida desses pacientes. Além de prevenir a perda de peso, desnutrição e deficiência de nutrientes, a 
alimentação adequada e saudável está associada a eficácia do sistema imunológico (COZER, 2016).

Assim como as crianças infectadas pelo HIV, as crianças expostas necessitam indispensavelmente do acompanhamento e orientação de um profissional da nutrição, já que os cuidados com o manuseio e preparo das fórmulas necessitam ser dialogados e acompanhados (TANAKA et al., 2015).

Nesse estudo a amostragem foi pequena, pelo fato de haver uma baixa prevalência de gestantes infectadas com HIV em Palmas. Em 2015, por exemplo, o número de mulheres vivendo com HIV que engravidaram foi de 14 (BRASIL, 2015).

Outra limitação do estudo foi 0 instrumento utilizado para coleta dos dados (Recordatório Alimentar de 24 horas) que possui limitado poder preditor de hábitos alimentares, visto que depende da memória do entrevistado, depende da capacidade de o entrevistador estabelecer uma boa comunicação e evitar a indução de respostas, além de a aplicação de um único Recordatório Alimentar não estimar a dieta habitual, pois pode ser que o dia avaliado seja um dia atípico (FISBERG; MARCHION; COLUCCI, 2009).

Para traçar um perfil dietético mais fidedigno à realidade dessas crianças, seria necessário um acompanhamento por um período de tempo maior, com a aplicação de outros inquéritos dietéticos.

\section{CONSIDERAÇOES FINAIS}

O perfil antropométrico das crianças deste estudo apresenta-se em maior parte adequado. Porém, observam-se crianças em risco de sobrepeso, o que pode ser agravado pela ingestão energética superior à necessidade, observada nesta população.

A maior parte das crianças, são filhas de mães jovens, com baixo grau de escolaridade, baixa renda familiar e renda per capita. Esse perfil materno denota uma população mais vulnerável a complicações relacionadas ao HIV.

Destaca-se a necessidade de mais estudos na população infantil exposta e vivendo com HIV/AIDS, buscando novas propostas para a individualização dos cuidados em saúde e nutrição desse grupo, de forma a promover melhorias no tratamento e na qualidade de vida.

\section{REFERÉNCIAS}

BRASIL. Ministério da Saúde. Secretaria de Vigilância em Saúde. Programa Nacional de DST e AIDS. Recomendações para terapia antirretroviral em crianças e adolescentes 
infectados pelo HIV: manual de bolso. Série manuais. Brasília: Ministério da Saúde, 2009. Disponível em: <http://www.aids.gov.br/sites/default/files/consenso pediatrico.pdf $>$ Acesso em: 23 nov. 2016.

BRASIL. Ministério da Saúde. Secretaria de Vigilância em Saúde. Secretaria de Atenção em Saúde. Guia prático de preparo de alimentos para crianças menores de 12 meses que não podem ser amamentadas. Brasília: Ministério da Saúde, 2004. Disponível em: < http://www.redeblh.fiocruz.br/media/guiamstaids.pdf > Acesso em: 24/11/2016.

BRASIL. Ministério da Saúde. Secretaria de Política de Saúde. Organização Pan Americana da Saúde. Guia alimentar para crianças menores de dois anos. Brasília: Ministério da Saúde, 2002. Disponível em: <http://www.opas.org.br/wp-content/uploads/2015/09/Guiaalimentar-criancas-2-anos.pdf> Acesso em: 24 nov. 2016.

BRASIL. Ministério da Saúde. Secretaria de Políticas de Saúde Departamento de Gestão de Políticas Estratégicas. Área Técnica de Saúde da Mulher. Gestação de Alto Risco. Manual Técnico. 3. ed. Brasília: Ministério da Saúde, 2000. Disponível em: $<$ https://www3.fmb.unesp.br/emv/pluginfile.php/1614/mod resource/content $/ 4 /$ manual\%20 \%20ar.pdf> Acesso em: 24 nov. 2016.BARROSO, L. M. M. et al. Cuidado materno aos filhos nascidos expostos ao HIV/AIDS. Rev. Rene. Fortaleza, v. 10, n. 4, out/dez 2009, p. 155-164.

CARVALHO, F. T. PICCININI, C. A. Sentimentos de mães portadoras de HIV/Aids em relação ao tratamento preventivo do bebê. Psico-USF (Impr.) [online]. 2008, vol.13, n.1, p. 75-83.

COZER, M. Estudo dos determinantes da insegurança alimentar em pacientes atendidos em um serviço de assistência especializada em HIV/AIDS. 2016. 111 f.. Dissertação (Mestrado em Gestão e Desenvolvimento Regional) - Universidade Estadual do Oeste do Paraná, Francisco Beltrão, 2017.

DIAS, R. M. et al. Crianças HIV positivas: características antropométricas e Sociodemográficas. Revista Paraense de Medicina, v. 26, n. 4, out/dez 2012. Disponível em: <http://files.bvs.br/upload/S/0101-5907/2012/v26n4/a3453.pdf>. Acesso em 30 nov. 2016.

DIETBOX. Software para Planejamento Dietético Dietbox versão 2.0. 2015. Disponível em: https://dietbox.me/pt-BR/.Acesso em: 16 nov. 2016.

EGASHIRA, E. M. et al. Diet'scharactheristics for childrenundertwoyearsof age in thecityof São Paulo, Brazil. In: Reunião da Sociedade Latino Americana de Investigação Pediátrica, 1988. Sociedade Latino Americana de Investigação Pediátrica, São Paulo 1998. p.47.

FILHO I, J. A Ética na Terapia Nutricional (Editorial).Revista Brasileira de Nutrição Clínica, v. 19, n. 2, 2004, p. III-IV.

FISBERG, R. M.; MARCHIONI, D. M. L.; COLUCCI, A. C. A. Avaliação do consumo alimentar e da ingestão de nutrientes na prática clínica. ArqBrasEndocrinolMetab., v. 53, n. 5, 2009. p. 617-24.

FREITAS, J.G. et al. Alimentação de crianças nascidas expostas ao Vírus da Imunodeficiência Humana.Texto Contexto Enferm., v. 23, n. 3, 2014. p. 617-25. 
GALVÃO, M. T. G. Condições sociodemográfica, materna e clínica de crianças expostas ao vírus da imunodeficiência humana. Rev Rene, v. 15, n. 1, 2014. p.78-88.

GOMES, M. A. T.; CABRAL, I. E.; SCHILKOWSKY, L. B. Crianças com HIV/AIDS de uma unidade ambulatorial pública: conhecendo seu perfil. RevSocBrasEnfermPediatr., v.4, n. 2, 2004. p. 55-68.

GRANGEIRO, A.; CASTANHEIRA, E. R.; NEMES, M. I. B. A re-emergência da epidemia de aids no Brasil: desafios e perspectivas para o seu enfrentamento. Interface, v. 19, n. 52, Jan-Mar 2015

MACHADO,M. M. T. et al. Condições sociodemográficas de crianças de zero a dois anos filhas de mães com HIV/AIDS Fortaleza, CE, Brasil. Revista Brasileira de Saúde Materno Infantil, v. 10, n. 3, 2010. p. 377-382.

REZENDE, F.A.C.; PEREIRA, R.J. Bases para o planejamento dietético. Palmas, TO: Editora da Universidade Federal do Tocantins/EDUFT, 2014. 240p.

SECRETARIA DE ESTADO DA SAÚDE DO TOCANTINS- SESAU. Diretoria Geral de Vigilância e Proteção à Saúde. Coordenação de DST/AIDS e hepatites virais. Sistema de Informação de Agravos de Notificação do Estado do Tocantins. Casos de AIDS em adultos, diagnosticados e residentes no Tocantins, de 2007 a 2012. Disponível em: $<$ http://www.saude.to.gov.br/index.php?option=com content\&view=article\&id=2474:diamundial-de-luta-contra-a-aids-tera-semana-de-programacao\&catid=45:noticias> Acesso em 04 fev. 2012.

SILVA, J.Práticas alimentares e anemia ferropriva nos primeiros dois anos de vida.2011. 26f. Monografia (Especialização em Atenção Básica em Saúde da Família) - Universidade Federal de Minas Gerais, Belo Horizonte - MG, 2011.

STATISTICAL PACKAGE FOR THE SOCIAL SCIENCES INCORPORATION. Statistical Package for the Social Sciences for Windows 20.0: base system user's guide. Chicago: SPSS Incorporation; 2011.

TADIELO, B.Z. et al. Morbidade e mortalidade de recém-nascidos em tratamento intensivo neonatal no sul do brasil.Rev. Soc. Bras. Enferm. Ped., v.13, n.1, Jul. 2013. p 7-12.

TANAKA, L. F.; LATORRE, M. R.; SILVA, A. M.; KONSTANTYNER, T. C., MENDES, E. C.; MARQUES, H. H. Poor diet quality among Brazilian adolescents with HIV/AIDS. J Pediatr, v. 91, n. 2, p. 152-159, 2015.

WORLD HEALTH ORGANIZATION- WHO. HIV andInfantFeeding: Guidelines for DecisionMakers. A Guide for Health Care Managers andSupervisors. 2.ed. Geneva (Switzerland): UNICEF/UNAIDS/WHO, 2003.Disponível em: <http://apps.who.int/iris/bitstream/10665/43864/1/9241591226.pdf>. Acesso em: 15 out. 2016.

WORLD HEALTH ORGANIZATION- WHO. Reference 2007: Growthreference 0-5 years. 2007. Disponível em: <http://www.who.int/childgrowth/standards/en/>.Acesso em 10 nov. 2016. 